\title{
Probing Device-relevant Defects in van der Waals Layered Materials
}

\section{Danielle Reifsnyder Hickey}

Pennsylvania State University, State College, Pennsylvania, United States

Many exciting van der Waals layered materials have been predicted and realized in recent years that could produce breakthrough performance in existing devices or will inspire the invention of new device configurations $[1,2]$. However, their promise is contingent upon being able to identify and engineer the structures of defects and interfaces.

The use of transmission electron microscopy (TEM) is ideal for such study because it can provide atomic detail and correlate it with larger defect structures. Additionally, in the TEM, samples can be investigated in plan-view or cross-section, making it possible to access a variety of orientations, including large areas of thin films and interfaces with substrates or stacks of layers in a device.

Here, I will discuss several examples of defect and interface structures in two-dimensionally layered materials for electronic and spintronic applications, and I will provide examples of how complementary modes in scanning and conventional TEM have provided much more structural information than a single type of measurement. First, I will discuss hidden defects such as interlayer rotations, defect phases at grain boundaries, and interfaces in topological insulators that have been grown by molecular beam epitaxy [36]. I will present examples of $\mathrm{Bi}_{2} \mathrm{Se}_{3}$ and $(\mathrm{Bi}, \mathrm{Sb})_{2} \mathrm{Te}_{3}$ on the substrates hexagonal boron nitride (h-BN), yttrium iron garnet, and $\mathrm{SrTiO}_{3}$. In these cases, defects identified by TEM can alter the physical properties of the thin films in ways that can impact device performance.

Next, I will discuss device-relevant defect structures in transition metal dichalcogenide (TMD) monolayers, such as $\mathrm{WS}_{2}$ grown on sapphire. Even epitaxially grown TMD films can have grain boundaries and defect arrays that can impact device performance. I will present recent results on grain boundaries and point defects that exist in single-orientation metal-organic chemical vapor depositionsynthesized films, showing the atomic structures and how they are ordered into large-area film features. For these examples, I will discuss how the defects are related to TMD growth conditions, as well as potential routes for engineering defects [7]. 
a
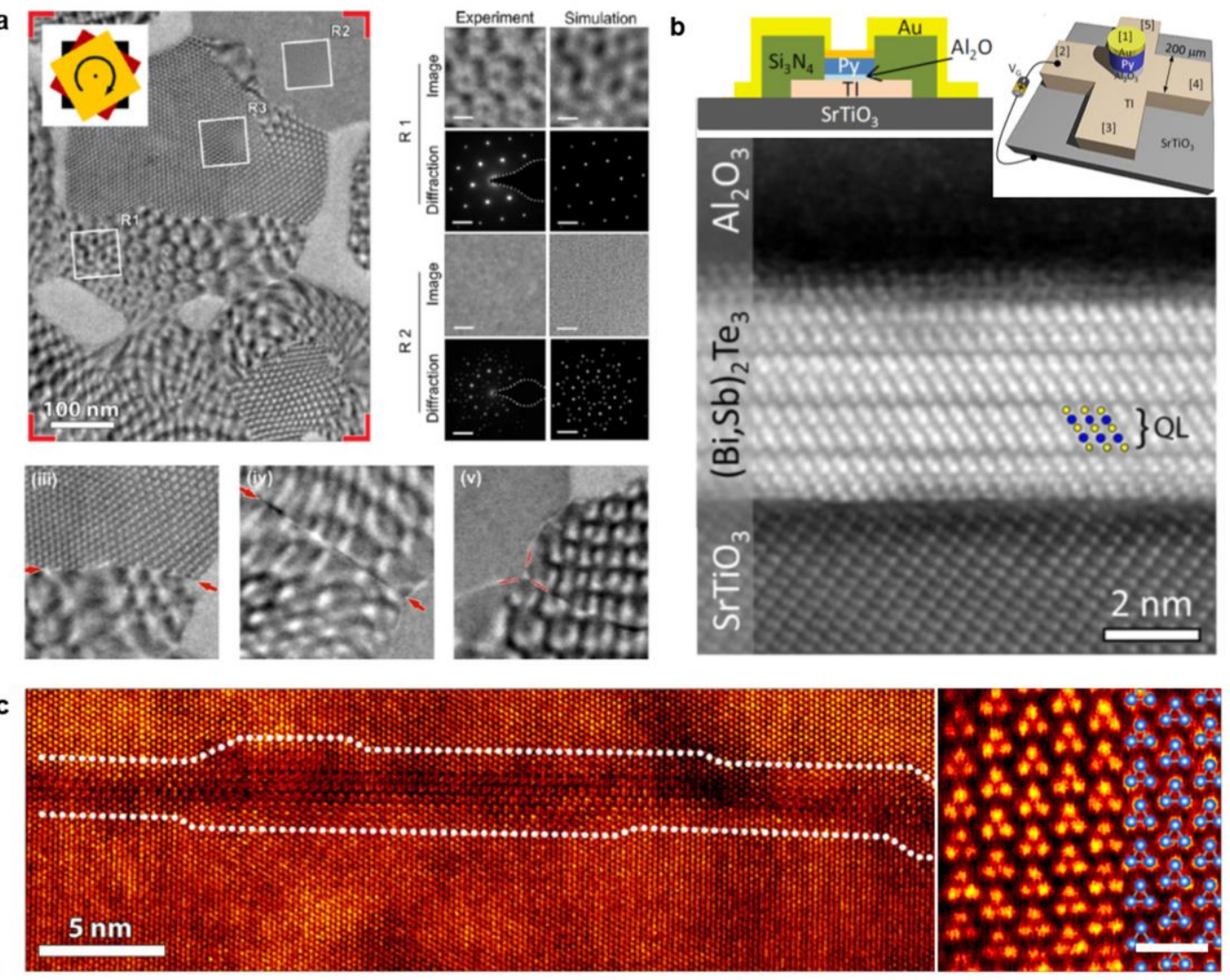

Figure 1. Examples of S/TEM characterization of defects in 2D heterostructures and devices. (a) Details of interlayer rotations in $(\mathrm{Bi}, \mathrm{Sb}) 2 \mathrm{Te} 3$ on $\mathrm{h}-\mathrm{BN}$, as shown by Moiré fringes and corresponding experimental and simulated electron diffraction patterns [3]. (b) Illustrations of a (Bi,Sb)2Te3/Al2O3/permalloy magnetic tunnel junction and a high-angle annular dark-field scanning TEM (HAADF-STEM) image of a cross section of the device [6]. (c) HAADF-STEM images of a Te phase at a grain boundary in (Bi,Sb)2Te3 [3]. Reprinted figures 2 and 4 with permission from [3] as follows: D. Reifsnyder Hickey, R.J. Wu, J.S. Lee, J.G. Azadani, R. Grassi, M. DC, J.-P. Wang, T. Low, N. Samarth, K. A. Mkhoyan, Phys. Rev. Mater., 4, 011201(R) (2020). Copyright (2020) by the American Physical Society. Reprinted figure 1 with permission from [6] as follows: J.S. Lee, A. Richardella, D. Reifsnyder Hickey, K. A. Mkhoyan, N. Samarth. Phys. Rev. B, 92, 155312 (2015). Copyright (2015) by the American Physical Society.

\section{References}

[1] S. Manzeli et al., Nat. Rev. Mater. 2 (2017) 17033.

[2] J.P. Heremans et al., Nat. Rev. Mater. 2 (2017) 17049.

[3] D. Reifsnyder Hickey et al., Phys. Rev. Mater. 4 (2020) 011201(R).

[4] D. Reifsnyder Hickey et al., Phys. Rev. Mater. 3 (2019) 061201(R).

[5] H. Wang et al. Phys. Rev. Lett. 117 (2016) 076601.

[6] J. S. Lee et al., Phys. Rev. B 92 (2015) 155312.

[7] The author gratefully acknowledges funding provided by C-SPIN, one of six STARnet program research centers, and by the 2D Crystal Consortium-Materials Innovation Platform (2DCC-MIP) under NSF cooperative agreement DMR-1539916. 